\title{
Evaluación de dietas suministradas al caracol Helix aspersa Müller
}

\author{
Evaluation of diets supplied to snail Helix aspersa Müller \\ Ruiz Hernández Ángela Kathyuska ${ }^{1}$, Salamanca Díaz Sandra Margot ${ }^{1}$ y \\ Hurtado Nery Víctor Libardo² \\ ${ }^{1} \mathrm{MVZ}$, Universidad de los Llanos y \\ ${ }^{2} \mathrm{MVZ}$, MSc, PhD, Docente Universidad de los Llanos \\ vhurtado@unillanos.edu.co
}

Recibido 10 de Mayo 2016, Aceptado 29 de Octubre 2016

\section{RESUMEN}

La cría de caracoles en Colombia es incipiente, sin embargo, las condiciones climáticas y oportunidades en el mercado externo son propicias para su desarrollo productivo y económico, debido a que la inversión es poca con un retorno rápido de capital; es por esto que el objetivo de esta investigación fue evaluar dietas con el fin de establecer el comportamiento del caracol Helix aspersa Müller, especie que prolifera en las zonas tropicales de Colombia. Los caracoles se confinaron en jaulas y fueron distribuidos en un diseño completamente al azar con cuatro tratamientos, seis replicaciones y 20 caracoles por cada una. Los tratamientos consistieron en: T1 = lechuga (Lactuca sativa); T2 = matarratón (Gliricidia sepium) (MA) y torta de soya (TS); T3 = harina de trigo (HT) y T4 = harina de arveja (HA), a todas las raciones se les adicionó carbonato de calcio. Se estimó: variación morfométrica, consumo, ganancia de peso, conversión, biomasa y tamaño de la concha. Se observó que el tipo de ingrediente influyó en la ganancia de peso, y la producción de biomasa por kg de alimento, siendo superior en T2 $(P<0.05)$ con relación a las demás dietas: T1: 4.57, T2: 6.06, T3: 5.77 y T4: 3.25 g; 0.264, 0.873, 0.693 y $0.428 \%$; mientras que la conversión alimenticia fue similar para T2 y T3: $3.78,1.14,1.44$ y 2.35 respectivamente. La longitud y ancho de concha no se afectaron ( $P>0.05)$ 1.66, 1.85, 1.74 y $1.66 \mathrm{~cm} ; 75,70,76.66$ y $82.50 \mathrm{~cm}$ respectivamente para $\mathrm{T} 1$ a $\mathrm{T} 4$. En conclusión, el nivel de aporte proteico de la dieta con G. sepium y torta de soya proporcionó ganancias de peso y 
conversiones alimenticias superiores en 25,5, y $46 \% ; 2.63,1.26$ y 2.06 veces respectivamente en comparación con T1, T3 y T4.

Palabras clave: Leguminosas, helicicultura, morfometría, moluscos.

\section{ABSTRACT}

The breeding of snails in Colombia is incipient, however climatic conditions and opportunities in the external market are propitious to their productive and economic development, because the investment is small with a quick return of capital; this is why the objective of this research was to evaluate diets in order to establish the behavior of the snail Helix aspersa Müller, species that proliferates in the tropics of Colombia. The snails were confined in cages and were distributed in a completely randomized design with four treatments, six replications and 20 snails each one. The treatments consisted of: $\mathrm{T} 1$ = lettuce (Lactuca sativa); $\mathrm{T} 2=$ Gliricidia sepium (MA) and soybean meal (TS); T3 = wheat flour (HT) and T4 = pea flour (HA), calcium carbonate was added to all rations. It was estimated: morphometric variation, consumption, weight gain, conversion, biomass and shell size. It was observed that the type of ingredient influenced the weight gain, And the production of biomass per $\mathrm{kg}$ of food, being higher in $\mathrm{T} 2(\mathrm{P}<0.05)$ in relation to the other diets: T1: 4.57, T2: 6.06, T3: 5.77 and T4: $3.25 \mathrm{~g} ; 0.264,0.873,0.693$ and $0.428 \%$; while the feed conversion was similar for T2 and T3: 3.78, 1.14, 1.44 y 2.35 respectively. Shell length and width were not affected $(P>0.05) 1.66,1.85,1.74$ and $1.66 \mathrm{~cm}$; $75,70,76.66$ and $82.50 \mathrm{~cm}$ respectively for $\mathrm{T} 1$ to $\mathrm{T} 4$. In conclusion, the level of dietary protein intake with $G$. sepium and soybean meal provided higher gains in weight and feed conversion in 25, 5, and 46\%; 2.63, 1.26 and 2.06 times respectively compared to $\mathrm{T} 1, \mathrm{~T} 3$ and $\mathrm{T} 4$.

Keywords: Legumes, heliciculture, morphometric, mollusc.

\section{RESUMO}

A criação de caracóis na Colômbia é incipiente, no entanto as condições meteorológicas e oportunidades em mercados estrangeiros são propícias para 0 
desenvolvimento produtivo e econômico, porque o investimento é pequeno, com um rápido retorno do capital; é por isso que o objetivo desta pesquisa foi avaliar dietas a fim de estabelecer o comportamento do caracol Helix aspersa Müller, espécie que prolifera em regiões tropicais da Colômbia. Os caracóis foram confinados em gaiolas e foram distribuídos em um delineamento experimental inteiramente casualizado, com quatro tratamentos, seis repetições e 20 caracóis cada um. Os tratamentos consistiram em: $\mathrm{T} 1$ = alface (Lactuca sativa); $\mathrm{T} 2=$ matarratón (Gliricidia sepium) (MA) e farelo de soja (TS); T3 = farinha de trigo (HT) e T4 = farinha de ervilha (HA), a todas as porções foi adicionado carbonato de cálcio. Foi estimado: morfométricas variação, consumo, ganho de peso, conversão de biomassa e tamanho da concha. Foi observado que o tipo de ingrediente influenciou o ganho de peso, ea produção de biomassa por $\mathrm{kg}$ de alimento, sendo superior em T2 $(\mathrm{P}<0.05)$ em relação a outras dietas: T1: 4.57, T2: 6.06, T3: 5.77 e T4: $3.25 \mathrm{~g}$; 0.264, 0.873, 0.693 e $0.428 \%$; enquanto que a conversão alimentar foi semelhante a T2 e T3: 3.78, 1.14, 1.44 e 2.35, respectivamente. O comprimento e largura da carapaça não foram afetados $(P>0.05)$ 1.66, 1.85, 1.74 e $1.66 \mathrm{~cm} ; 75$, $70,76.66$ e $82.50 \mathrm{~cm}$ respectivamente para T1 a T4. Em conclusão, o nível de ingestão de proteínas da dieta com $G$. sepium e farelo de soja deu os ganhos de peso e conversões alimentares acima de 25, 5, e 46\%; 2.63, 1.26 e 2.06 vezes, respectivamente, em comparação com T1, T3 e T4.

Palavras-chave: Leguminosas, helicicultura, morfometria, moluscos.

\section{INTRODUCCIÓN}

Los caracoles Helix aspersa Müller, son moluscos terrestres comunes en las zonas tropicales, su concha mide entre 28 y $32 \mathrm{~mm}$ de diámetro y son de color marrón con manchas café oscuro, presentan dimorfismo sexual y se reproducen sexualmente, alcanzando su madurez a los 6 meses de edad. Es ovíparo y deposita sus huevos dentro de una cámara que hace dentro de la tierra a una profundidad entre 5 a $10 \mathrm{~cm}$, se clasifican por sus hábitos alimenticios como herbívoros, siendo eficientes convertidores de energía, por lo tanto los alimentos a utilizar son de bajo costo y fácil adquisición (Montoya et al., 2008). 
Los caracoles en general, requieren suelos calizos, bien sean selváticos o con escasa vegetación, siendo la humedad indispensable puesto que ésta regula su actividad, requiere humedad relativa del $75-90 \%$, puesto que mayores y menores valores hacen disminuir sus funciones vitales. La temperatura óptima para la especie fluctúa entre 15 a $25^{\circ} \mathrm{C}$, siendo la ideal $20^{\circ} \mathrm{C}$, por lo que temperaturas superiores o inferiores e este valor también limitan su actividad (Fernández, 2013).

El fotoperíodo influye en gran medida en su actividad vital y reproductiva, por lo que en cautiverio se utiliza una luminosidad baja (más de 14 horas en penumbra) en los lotes de caracoles en reproducción; caso opuesto a lo sucedido con los lotes en engorde los cuales requieren de mayores niveles de luz (hasta 16 horas de luz). El viento por sus efectos sobre la evaporación tegumentaria y, por lo tanto, sobre su hidratación corporal tiene también un efecto desfavorable cuando se adquiere una velocidad excesiva, de ahí que los caracoles busquen lugares protegidos de las fuertes corrientes de aire (Cabrera et al., 2013).

La especie originalmente encontrada en jardines, se encuentra también en ambientes seminaturales como cultivos, parques con condiciones de humedad y sombra. Es originaria de Europa occidental donde culturalmente se consume su carne, constituyéndose en un plato costoso, y se introdujo a algunos países de Sur América (Argentina, Brasil, Colombia, Chile y Ecuador) para desarrollar su producción y suplir la demanda de los países consumidores por tradición (España, Francia e Italia) (Díaz et al., 2007).

Sus productos de secreción han sido investigados y caracterizada su composición química (Svendsen et al., 2016), enfocándose en sus facultades nutricionales (Zarai et al., 2016), antitumorales (Romeila et al., 2016), antihipertensivas (Cudennec et al., 2016) y otros. También se han estudiado las condiciones reproductivas del caracol en función del ambiente (Cabrera et al., 2013).

Por otro lado como fuente de proteína ha sido evaluado la sobrevivencia, y su calidad de la carne (González et al., 2013), y también se ha evaluado el efecto del 
tratamiento térmico sobre la firmeza, sabor de la carne y aceptabilidad general de la sopa de caracol enlatada (Díaz, 2014).

Los zoocriaderos en Colombia están distribuidos principalmente en la región Andina, los cuales se han desarrollado y diseñado bajo diferentes escalas y esquemas de producción, registrándose un total de 45 en todo el país (Arrieta y Estupiñan, 2010). La helicicultura ha sido promocionada por diferentes grupos y asociaciones como una alternativa productiva con baja inversión y retorno del capital invertido en un corto tiempo (Tabla 1). Se han logrado identificar mercados potenciales para la carne de caracol cuya viabilidad depende de la demanda a nivel internacional y del cumplimiento con los requerimientos ambientales y sanitarios exigidos en los países compradores, puesto que la demanda de carne de caracol en el país es casi nulo, lo cual contrasta con el alto consumo reportado en Italia, España y Francia, en este último país existe una demanda anual de más de 100.000 toneladas (Alaguna y Amador, 2008).

Tabla 1. Indicadores zootécnicos y económicos para la helicicultura en Colombia

Producción/caracol/año

Postura anual

Área necesaria por pie de cría

Producción/ $\mathrm{m}^{2} / a n ̃ o$

Precio de venta $\mathrm{kg}$ de caracol vivo en Colombia

Precio de venta $\mathrm{kg}$ de caracol vivo en Mercado Internacional

Valor de cada reproductor seleccionado como pie de cría

Costo de construcción del invernadero por m2
192 caracoles viables $(1.5 \mathrm{~kg})$

320 huevos

$3 \mathrm{~m}^{2}$

$1.8-2 \mathrm{~kg}$

$\$ 5.000-7.000$

US $\$ 3-7$

$\$ 300-350$

$\$ 1500-2000$

Fuente: FEDECOHEL, (2016).

Además de FEDECOHEL (Federación Colombiana de Helicicultura), los productores han constituido varias asociaciones helicícolas como ASOCOHÉLIX, COPOHÉLIX, ASOPEC, INDUAGROCOL, INTRAGROCOL, COFEDERACOL, FEDECOHEL, FUNCOLSA, DHELIEXPORT DE COLOMBIA LTDA.,COHECOL, y ANAYACOLY, enfocando sus objetivos están enfocados principalmente en el 
desarrollo de esta industria y a promover su consumo de carne en el país (Mejia et al., 2008).

En Colombia se presentan ventajas ecológicas para la producción de caracol, dadas las condiciones climáticas sin estaciones (FINAGRO, 2013), lo que convierte a la helicicultura en un negocio muy atractivo para los inversionistas debido a su positiva proyección en el futuro y su alta rentabilidad (Díaz et al., 2007). El Instituto Colombiano Agropecuario (ICA, 2010), estableció los requisitos para el registro y las condiciones sanitarias para los predios productores de caracoles del género Helix cuya carne, productos cárnicos comestibles sean destinados al consumo humano.

La especie no solo implica producción de carne, sino que también ha sido explotada con fines cosméticos, adicionalmente ha sido utilizada como biomodelo como indicador en algunas investigaciones de tipo ambiental y fisiológico (Regoli et al., 2006). Según FINAGRO, (2013) el sistema productivo de Helix aspersa se realiza en recintos cerrados o cría intensiva en colgadores plásticos verticales permitiendo obtener mayor aprovechamiento de la superficie del recinto, el ambiente debe ser controlado con temperatura de 15 a $21^{\circ} \mathrm{C}$, humedad de $70-80 \%$ y fotoperiodo de 12 horas luz.

La cría del caracol terrestre del género Helix en régimen de cautividad se denomina helicicultura, es una fuente importante de proteína de origen animal para la alimentación humana, principalmente en la cultura occidental, donde los países de mayor consumo de Helix aspersa son Francia, Italia, Alemania, Suiza y España donde se registra un auge en esta industria (Aragón et al., 2016), en México es una actividad relativamente nueva, con antecedentes de producción en pequeñas granjas (Bermúdez y Vázquez, 2016).

La alimentación, como en cualquier especie animal debe atender los requerimientos nutricionales, en el caso del Helix aspersa estas necesidades no se han establecido totalmente. Sin embargo, Soares et al., (1999) recomiendan $18.28 \%$ de proteína y $5 \%$ de calcio para mejorar las características de rendimiento 
(Cayambe, 2012; Hayashi et al., 2005), aunque es necesario balancear las dietas para que suplan adecuadamente los requerimientos de proteína y energía de acuerdo con la fase desarrollo, estado fisiológico, manejo y nivel de producción deseado, de este modo, Perea et al., (2008) consideran que no hay raciones adecuadas para la cría comercial de caracoles terrestres porque se desconocen las algunos procesos metabólicos y fisiológicos que son importantes para determinar el aprovechamiento de los nutrientes en el organismo de esta especie de caracol.

Por lo anterior, el objetivo de este trabajo fue el de evaluar el efecto de dietas elaboradas con diferentes ingredientes y contenido nutricional, utilizando materias primas de origen vegetal: lechuga (Lactuca sativa), matarratón (Gliricidia sepium) (MA) y torta de soya (TS), harina de trigo y harina de arveja (HT y HA) (Tabla 2) para observar el comportamiento de los parámetros productivos de los caracoles Helix aspersa Müller.

Tabla 2. Composición de las materias primas utilizadas en las raciones para caracoles

\begin{tabular}{lccccc}
\hline Materia prima & $\begin{array}{c}\text { Arveja } \\
\text { verde }\end{array}$ & Lechuga & Matarratón & $\begin{array}{c}\text { Harina } \\
\text { de trigo }\end{array}$ & $\begin{array}{c}\text { Harina de } \\
\text { torta de soya }\end{array}$ \\
\hline Materia seca & 34 & 10 & 28.5 & 87 & 92 \\
Proteína & 8.2 & 1.2 & 20 & 12.5 & 48.3 \\
Calcio & 0.036 & 0.044 & 1.38 & 0.050 & 3.01 \\
$\begin{array}{l}\text { Energía Bruta / kg } \\
\text { (Kjcal de MS }\end{array}$ & 3200 & 1800 & 3050 & 3480 & 3250 \\
\hline
\end{tabular}

Fuente: Greenfield y Southgate, (2006) y (Araque et al.. 2006)

\section{METODOLOGÍA}

El trabajo fue realizado en Cumaral, Meta, municipio ubicado a $452 \mathrm{msnm}$ de altitud; con temperaturas promedio de $27^{\circ} \mathrm{C}$, humedad relativa de $84 \%$ en los meses de mayor precipitación y valores cercanos al $77 \%$ en los meses secos. La precipitación varía de 3500 a 2500 mm/año, la luminosidad de 4563 h/luz/año (IDEAM, 2014). 
Los caracoles se confinaron en jaulas de $40 \times 60 \mathrm{~cm}$, y fueron distribuidos en un diseño experimental completamente al azar en cuatro tratamientos, seis repeticiones y 20 caracoles por repetición (Figura 1). Para la recolección de la información se realizó un registro quincenal de la variación morfométrica, consumo de alimento, ganancia de peso, conversión, biomasa y tamaño de la concha

La fase experimental tuvo duración de tres meses, para el manejo diario, se utilizaron atomizadores para diluir los productos a base de yodo e insecticidas para desinfectar superficies y medio ambiente y esponja de aseo para los animales, además un termómetro para estimar cambios de la temperatura y por lo tanto hacer el manejo adecuado de las tapas de las jaulas o caracolarios (Figura 1). Las raciones fueron suministradas en platos desechables distribuidas uniformemente, y para proveer el agua se instaló una esponja humedecida el interior de cada jaula y se rociaron varias veces al día para mantener la humedad del medio ambiente, factor que tiene gran importancia en desarrollo y crecimiento de los caracoles.

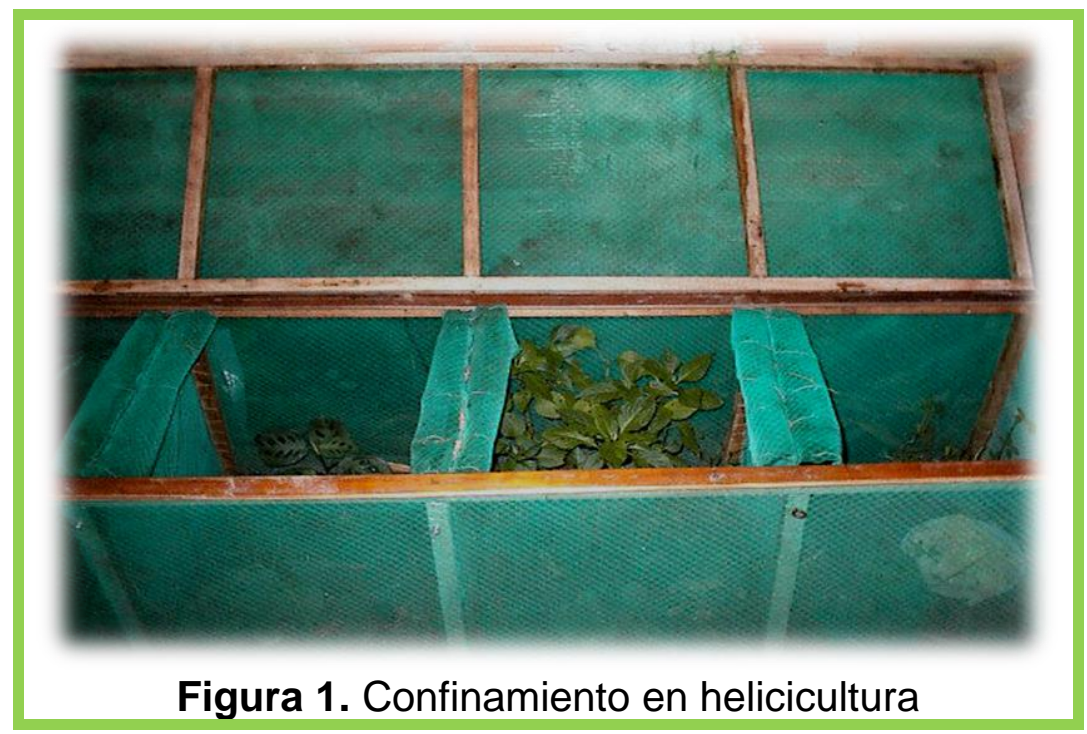

Las dietas experimentales se suministraron a voluntad en platos plásticos y estaban constituidas por lechuga fresca (T1); matarratón 50\% y torta de soya $50 \%$ (T2); harina de trigo 100\% (T3); harina de arveja 50\% y matarratón 50\% (T4), a todas las raciones de les adicionó carbonato de calcio (Tabla 3). 
El modelo estadístico utilizado para el análisis de los datos recopilados fue el siguiente: $\gamma i j=\mu+\tau i+\varepsilon$, siendo: $\mu$ : el valor medio de todos los datos independientemente del factor, $\tau i$ : es el efecto que tiene la dieta sobre la variable de respuesta, y $\varepsilon$ : el error experimental. Los datos fueron sometidos a análisis de varianza y comparación de medias por la prueba de Tukey a $0.5 \%$ de probabilidad.

Tabla 3. Consumo de nutrientes en las diferentes dietas

\begin{tabular}{ccccc}
\hline Consumo & T1 & T2 & T3 & T4 \\
\hline Materia seca total $(\mathrm{g})$ & 17.31 & 6.94 & 8.34 & 7.64 \\
Proteína $(\mathrm{g})$ & 0.2078 & 2.1618 & 1.0425 & 1.3446 \\
Energía Bruta Kical & 31.158 & 22.208 & 25.437 & 26.587 \\
\hline
\end{tabular}

Lechuga (T1); matarratón y torta de soya (T2); harina de trigo (T3) y harina de arveja y matarratón (T4). Cálculos con base a los valores reportados Greenfield y Southgate, (2006) y Araque et al., (2006).

\section{RESULTADOS Y DISCUSIÓN}

Se observó que la alimentación con dietas que contenían lechuga y harina de arvejas afectó $(\mathrm{P}<0.05)$ la ganancia de peso comparado con las dietas conteniendo matarratón, cuyo menor valor se obtuvo con la dieta basada en harina de arveja. La misma situación se constató con el rendimiento, los tratamientos con vegetales frescos y harina de arveja disminuyeron $(P<0.05)$ el rendimiento, el cual expresó su mayor rendimiento en el tratamiento con matarratón y torta de soya, seguido de la dieta con harina de trigo, esto explicado posiblemente por el hecho que los vegetales verdes contienen mucha agua con poco aporte de nutrientes, como el T1 a base de lechuga cuyo contenido de nutrientes está representado por energía (91.2\%) el cual mostró el menor rendimiento en comparación con las otras dietas, indicando que los caracoles requieren una dieta más equilibrada en términos de proporción proteína energía, lo cual se evidenció en el T2 a base de matarratón y torta de soya lo cual aportó aproximadamente $40 \%$ energía y $60 \%$ proteína (Figura 2); podría deducirse que dietas con proporciones de energía superiores a $40 \%$ afectan negativamente el rendimiento de los caracoles y 
términos de ganancia de peso, y mientras mayor sea su nivel en mayor medida se verá afectado (Tabla 4).

Tabla 4. Efecto del tipo de alimento sobre los parámetros productivos y morfométricos de Helix aspersa

\begin{tabular}{|c|c|c|c|c|}
\hline Variables & $\begin{array}{c}\text { Vegetales } \\
\text { Frescos }\end{array}$ & $\begin{array}{c}\text { Matarratón } \\
+ \text { Torta de } \\
\text { soya }\end{array}$ & $\begin{array}{c}\text { Harina de } \\
\text { Trigo + Torta } \\
\text { de soya }\end{array}$ & $\begin{array}{c}\text { Harina de } \\
\text { Arveja + } \\
\text { Matarratón }\end{array}$ \\
\hline Peso inicial. $\mathrm{g}$ & 1.66 & 1.75 & 1.69 & 1.82 \\
\hline Peso final. $g$ & 6.24 & 7.82 & 7.47 & 5.07 \\
\hline Consumo total. $\mathrm{g}$ & 17.31 & 6.94 & 8.34 & 7.64 \\
\hline Ganancia de peso. g & $4.57^{b}$ & $6.06^{a}$ & $5.77^{\mathrm{ab}}$ & $3.25^{c}$ \\
\hline Conversión alimenticia & $3.78^{a}$ & $1.14^{b}$ & $1.44^{b}$ & $2.35^{\mathrm{ab}}$ \\
\hline Biomasa / kg de alimento & $0.264^{c}$ & $0.874^{a}$ & $0.693^{\mathrm{ab}}$ & $0.428^{c}$ \\
\hline \multicolumn{5}{|l|}{ Morfometría } \\
\hline Longitud de la concha. $\mathrm{cm}$ & 2.73 & 2.94 & 2.89 & 2.71 \\
\hline Ancho de la concha. $\mathrm{cm}$ & 1.66 & 1.85 & 1.74 & 1.66 \\
\hline
\end{tabular}

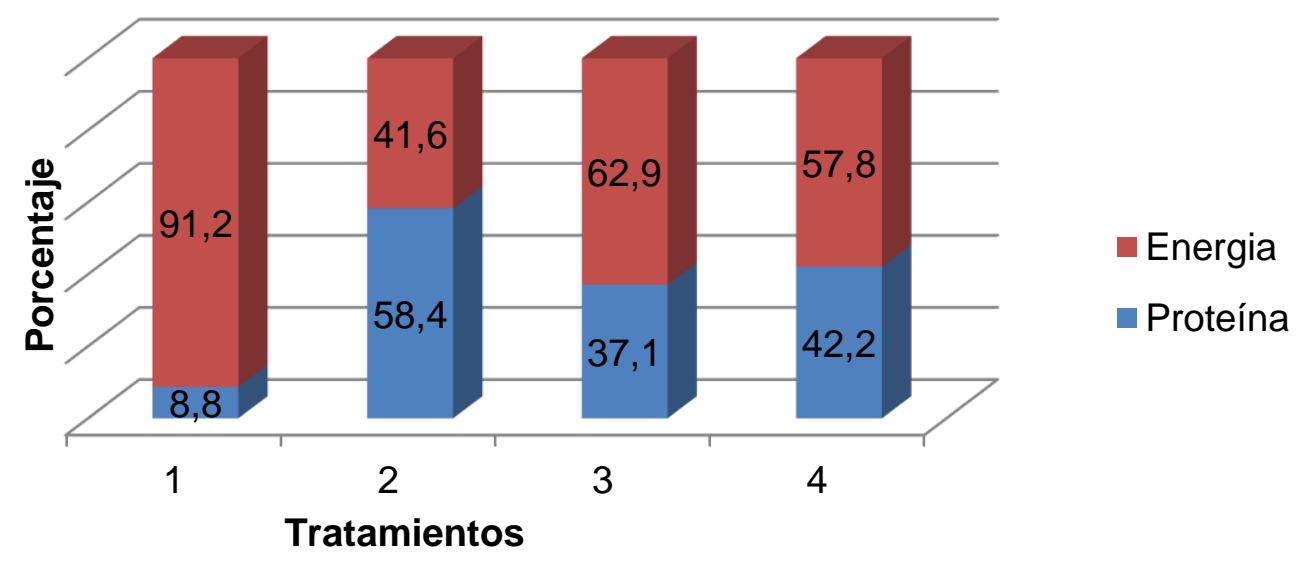

T1: Lechuga, T2: Matarratón + Torta de soya, T3: Harina de trigo, T4: Matarratón + Harina de arveja

Figura 2. Proporción (\%) de proteína y energía 
La mejor conversión $(P<0.05)$ se obtuvo en los tratamientos con dietas basadas en matarratón + torta de soya (T2) y harina de trigo + torta de soya (T3), entretanto la peor conversión fue obtenida con la dieta conteniendo harina de arveja + matarratón (T4).

La ganancia de peso obtenida en este trabajo fue inferior a la reportada por (Soares et al., 1999), quienes además obtuvieron mejor conversión alimenticia, explicando los resultados por el nivel de proteína suministrado. El consumo de alimento y la ganancia de peso fue inferior al obtenido por Machado et al., (2008). Acevedo et al., (2014) evaluaron la inclusión de diferentes tipos de harinas de Tithonia diversifolia y Gliricidia sepium en la alimentación de Helix aspersa Muller en fase de levante-ceba sobre la ganancia de peso y mortalidad, encontrando la mayor ganancia de peso lo presentó con inclusión a base de harina de matarratón, seguido del tratamiento con inclusión de botón de oro, además observaron una alta mortalidad en los tres tratamientos.

Por otro lado Cuéllar et al., (1987) estudiaron el efecto de la densidad sobre el tamaño del Helix aspersa Müller durante la primera fase de crecimiento (hasta 1.2 $\mathrm{cm}$ de longitud), utilizando 18 niveles de densidad entre 600 a 11100 animales $/ \mathrm{m}^{2}$,encontrando que la densidad óptima, en primera edad, oscila entre 600 y 4300 animales $/ \mathrm{m}^{2}$, y en el presente trabajo estuvo lejos del límite inferior encontrando buenos rendimientos productivos.

En el presente trabajo, las variables relacionadas con la morfometría no fueron influenciadas $(P>0.05)$ por la composición de la dieta, lo que sugiere que los niveles de calcio en la dieta fueron adecuados para atender los requerimientos de este nutriente para la deposición en la concha, puesto que los niveles de carbonato de calcio afectan directamente el crecimiento y mortalidad de los caracoles (Mayoral et al., 2004).

Por otra parte Perea et al., (2008) plantearon que el humus es importante en la dieta de Helix aspersa y de otras especies de caracoles comestibles aunque su contribución nutricional es poco conocida, por lo tanto evaluaron un suplemento 
con humus sobre el crecimiento, eficiencia nutricional y digestibilidad del caracol $H$. aspersa durante la fase juvenil, encontrando que cuando los caracoles crecen en presencia de humus lo consumen regularmente y aumentan la velocidad de crecimiento $(P<0.01)$. Además, el humus también aumenta la disponibilidad de los nutrientes orgánicos de la dieta, mejorando la digestibilidad de la materia seca, proteína cruda, fibra detergente neutro y fibra detergente ácido $(P<0.001)$. Por tanto, se mejoró la razón de conversión alimenticia y la razón de eficiencia proteica $(P<0.01)$.

En términos sanitarios Moncada et al., (2007) realizaron un diagnostico en diversos zoocriaderos helicícolas en Colombia, en el cual determinaron que los principales agentes bacterianos que afectan al caracol Helix aspersa en el país son: Salmonella sp., Staphylococcus aureus, Hafnia alvei, Pseudomonas sp., Streptococcus sp., Aeromonas hydrophila, Arcanobacterium sp., Corynebacterium sp., Escherichia coli y Hafnia alvei; hongos Geotrichum sp., Penicillium sp., y Aspergillus sp; y parásitos de las familias Strongyloididae, Oxiuridae, Riccardoella limacum y Nyctotheridae.

En otra especie de caracol (Pomacea maculata), Marina y Yalta, (2014) realizaron un ensayo con alimento balanceado tipo pellets con $30 \%$ de proteína bruta con inclusión de harina de cáscara de huevo al 1, 2 y 3\%; se utilizó además, para la elaboración de las raciones: harina de pescado, harina de torta de soya, harina de maíz, polvillo de arroz, sal y complejo vitamínico. Los resultados muestran que existen diferencias significativas en peso total, peso del caparazón con el opérculo y peso del cuerpo blando, como también en el ancho de caparazón, del mismo modo en resistencia a la fractura existió diferencia significativa. También se observó que el crecimiento, la resistencia a la fractura y la sobrevivencia fueron afectados por factores como: la estrategia de alimentación, la presentación del alimento y la calidad del agua, finalmente concluyen que la inclusión de harina de cáscara de huevo en raciones para caracoles influye positivamente en su crecimiento y fortalecimiento del caparazón. 
En consideración final Arditi et al., (2003) resaltan que el mercado objetivo de esta industria está compuesto por las importaciones de España, Italia, Grecia y Estado Unidos, cuyo mercado tiene un volumen de 13000 ton/año con un crecimiento estimado entre 450-2650 ton/año, cuya principal ventaja es que la demanda se encuentra insatisfecha, planteando una buena oportunidad productiva para el país

\section{CONCLUSIONES}

El nivel de aporte proteico de la dieta con matarratón y torta de soya, proporciona los mejores resultados de conversión alimenticia y morfometría en caracoles Helix aspersa, cuya proporción (\%) de proteína energía es aproximadamente 60:40.

Los niveles de proteína y calcio en las dietas fueron adecuados para atender los requerimientos de los caracoles para crecimiento y deposición de calcio en la concha.

\section{REFERENCIAS BIBLIOGRÁFICAS}

1. Acevedo C.M., Zuluaga Z.M., Montoya H.J.I. Efecto de la inclusión de diferentes tipos de harinas Botón de Oro (Tithonia diversifolia) y Matarratón (Gliricidia sepium) en la alimentación del caracol de tierra (Helix aspersa Muller) en fase de levante-ceba sobre la ganancia de peso y mortalidad, Unisarc, 2014.

2. Alaguna M.I., Amador R.S. Estudio de mercadeo para la explotación de caracol "Helix aspersa" a España, Administrador de Empresas Agropecuarias. Facultad de Administración de Empresas Agropecuarias, Universidad de la Salle, Bogotá, Colombia. 91 p. 2008.

3. Aragón M., Angón E., Rodríguez J., Barba C., Perea J., López M., Cabello A. Viabilidad del caracol terrestre (Cantareus aspersus) durante la época de descanso. Archivos de Zootecnia. 65, (251): 417-419. 2016.

4. Araque C., Quijada T., Páez L., Sánchez-Ruiz A., Espinoza F. Bromatología del mataratón (Gliricidia sepium) a diferentes edades de corte en Urachiche, estado Yaracuy, Venezuela. Zootecnia Tropical. 24, (4): 393-399. 2006.

5. Arditi A., Rodríguez D., Villar R. Plan de negocios para la cría, procesamiento y comercialización de caracoles Helix aspersa. Tesina, Universidad Centro de Estudios. 2003.

6. Arrieta S.M., Estupiñan G.A. Creación y puesta en marcha de una empresa de cultivo de caracoles para la obtención de baba de caracol, Ingeniero Industrial. Facultad de Ingeniería Físico - Mecánicas, Universidad Industrial de Santander, Bucaramanga, Colombia. 366 p. 2010.

7. Bermúdez R., Vázquez R. Implementación de un modelo lean para el cultivo y cría intensiva de la Helix aspersa. UPIICSA. 2, (1): 51-63. 2016. 
8. Cabrera H.Á.F., Cabrera L.A.M., García M.A.R. Efecto de las condiciones microclimáticas en el comportamiento reproductivo del Helix aspersa. Scientia Agropecuaria. 4, (4): 293-301. 2013.

9. Cayambe M.A. Evaluación del comportamiento productivo y reproductivo y reproductivo de caracoles (Helix aspersa) criados en parques de engorde, Ingeniero Zootecnista. Facultad de Ciencias Pecuarias, Escuela Superior Politécnica de Chimborazo, 2012.

10. Cudennec B., Violle N., Chataigné G., Drevet P., Bisson J.-F., Dhulster P., Ravallec R. Evidence for an antihypertensive effect of a land snail (Helix aspersa) by-product hydrolysate-Identification of involved peptides. Journal of Functional Foods. 22, 602-611. 2016.

11. Cuéllar L., García T.P., Fontanillas J., Sotillo J. Estadísticos reproductivos de Helix aspersa, L. var. maxima en ambiente controlado. Anales de Veterinaria de Murcia. 3: 99-102. 1987.

12. Díaz C.G.N. Efecto del tratamiento térmico sobre la firmeza, sabor de la carne y aceptabilidad general de sopa de caracol (Helix Aspersa) enlatada, Ingeniero en Industrias alimentarias. Facultad de Ciencias Agrarias, Universidad Privada Antenor Orrego, 2014.

13. Díaz J.L., Aguirre J.C., Mejía G.S., Martínez E. Reproducción y genética del caracol terrestre "Helix asper". Revista CES Medicina Veterinaria y Zootecnia. 2, (2): 78-88. 2007.

14. FEDECOHEL, Federación Colombiana de Helicicultura, Cría de caracol (Helix aspersa Muller). 2016. Recuperado 26 Marzo 2016. Disponible En: http://abc.finkeros.com/cria-de-caracol-helix-aspersa-muller/

15. Fernández $\mathrm{H}$. Efecto de la humedad relativa sobre la reproducción del caracol de tierra (Helix aspersa Müller). Avances en Investigación Agropecuaria. 17, (2): 97104. 2013.

16. FINAGRO, Fondo para el Financiamiento del sector agropecuario, Helicicultura. 2013. Recuperado 17 Enero 2013. Disponible En: http://www.finagro.com.co/html/i portals/index.php?p origin=internal\&p name=co ntent\&p id=Ml-16423\&p options=\#MUNDO

17. González O.M., Vieites C.M., Cossu M.E. Sobrevivencia, porcentaje de merma y calidad de carne de caracoles Hélix aspersa terminados en sistema abierto y almacenados en frío. Revista Argentina de Producción Animal. 28, (3): 227-233. 2013.

18. Greenfield H., Southgate D. Datos de composición de alimentos. Obtención, gestión y utilización. Burlingame BA, Charrondiere UR, editores. FAO, INFOODS, Roma. 330 p. 2006.

19. Hayashi C., Soares C., Matsushita M., Goncalves S. Teores de cálcio em rações para o escargot francês Helix aspersa maxima em fase de crescimento. Acta Scientarum. Biological Sciences. 27, (1): 57-61. 2005.

20. ICA, Instituto Colombiano Agropecuario, Resolución 1464 de 2010. Ministerio de Agricultura y Desarrollo Rural. Colombia, 2010.

21. IDEAM, Instituto de Hidrología, Meteorología y Estudios Ambientales, Información Histórica, Climatografía de las principales ciudades, Cartas Climatológicas Medidas Mensuales, Aeropuerto Vanguardia. 2014. Recuperado 25 Septiembre 2015. Disponible En: http://bart.ideam.gov.co/cliciu/villavo/tabla.htm

22. Machado J., Araújo R., Calheiros D., Deleito C., Mendes E., Baroni F. Influência da levedura Saccharomyces cerevisiae (Meyen ex Hansen, 1883) no 
desenvolvimento de escargots gros gris (Helix aspersa maxima). Revista Brasileira de Agrociência. 14, (1): 101-108. 2008.

23. Marina J.D., Yalta J.E. Influencia del alimento balanceado con tres niveles de inclusión de harina de cáscara de huevo, en el crecimiento y en la resistencia a la fractura de la concha del "churo", Pomacea maculata (ampullaridae), cultivados en jaulas, en el centro de investigación, experimentación y enseñanza, Piscigranja Quistococha, Biólogo Acuicultor. Facultad de Ciencias Biológicas, Universidad Nacional de la Amazonia Peruana, lquitos, Perú. 67 p. 2014.

24. Mayoral A., Perea J., Delgado M., Martín R., Acero R., García A. Efecto de la adición de carbonato cálcico en la dieta de" helix aspersa müller". Archivos de Zootecnia. 53, (204): 407-410. 2004.

25. Mejia J.D., García L., Juan M., Eslava O. Plan de negocios, Cría y comercialización de caracoles a través de la técnica de helicicultura, Especialista en Finanzas y Negocios Internacionales. Facultad de Ciencias Económicas y Administrativas, Universidad de la Sabana, Chía. Colombia. 210 p. 2008.

26. Moncada R.F.A., Veloza M.P.A., Rodríguez M.G., Reyes H.L. Diagnóstico sanitario de diversos zoocriaderos helicícolas en Colombia: determinación de los principales agentes patógenos que afectan el caracol Helix aspersa (OF Muller, 1774) en cada etapa de su ciclo biológico. Revista de Medicina Veterinaria. (14): 17-35. 2007.

27. Montoya J.I., Villegas C., Vanegas J.D. Evaluación del comportamiento reproductivo del caracol de tierra (Helix aspersa muller) bajo cuatro sistemas de alimentación diferentes. Investigaciones UNISARC. 6, (1): 8-16. 2008.

28. Perea J., García A., Acero R., Peña F., Gómez G. Efecto de un suplemento con humus sobre el crecimiento, digestibilidad y eficiencia nutricional en el caracol Helix aspera juvenil. Agrociencia. 42, 165-171. 2008.

29. Regoli F., Gorbi S., Fattorini D., Tedesco S., Notti A., Machella N., Bocchetti R., Benedetti M., Piva F. Use of the land snail Helix aspersa as sentinel organism for monitoring ecotoxicologic effects of urban pollution: an integrated approach. Environ Health Perspect. 114, (1): 63-9. 2006.

30. Romeila M., Dalila N., Meriem R., Frank A. Comparative study of two helix aspersa extracts on tumor cell lines (hut-78 and seax) proliferation and $\mathrm{mmp}-9$ secretion. International Journal of Pharmacy and Pharmaceutical Sciences. 8, (11). 2016.

31. Soares C., Hayashi C., Goncalves G., Nagae M., Boscolo W. Exigência de proteína para o caracol gigante gigante (Achatina áulica) em fase de crescimento. Acta Scientiarum. 21, (3): 683-686. 1999.

32. Svendsen A.H., Larsen S.K., Ranum K., Vélez J.F.M. Chemical analysis of the composition of stress induced mucus from Helix aspersa, 2016.

33. Zarai Z., Balti R., Sila A., Ali Y.B., Gargouri Y. Helix aspersa gelatin as an emulsifier and emulsion stabilizer: functional properties and effects on pancreatic lipolysis. Food \& function. 7, (1): 326-336. 2016. 